\title{
"EVALUACIÓN PRELIMINAR DE LA CALIDAD DEL AGUA PARA CONSUMO HUMANO DE LA POBLACIÓN DEL DISTRITO DE SAMA Y ALTERNATIVAS DE TRATAMIENTO"
}

Responsable: Dr. Alberto B. Quispe Cohaila Miembro: Ing. Victor Malpartida Arrieta Miembro: Mgr. Biologo Juan Franco

\begin{abstract}
RESUMEN
El presente trabajo fue desarrollado con el fin de estudiar los problemas ambientales en el distrito de Sama, en especial la calidad de agua que consume la población de Las Yaras, los anexos de Buena Vista y Miraflores. El trabajo muestra los problemas de salud que causa el arsénico en la población, la dosificación promedio teórica que ingiere una persona, la utilización de aluminio reciclado para obtener sulfato de aluminio como coagulante y precipitador del arsénico y la remoción obtenida por este componente. También se propone un plan de acción para la zona con el fin de mejorar la calidad ambiental.
\end{abstract}

\begin{abstract}
he present task has developed in order to study environment's problems in Sama Region, especially water's quality that people who live in the Yaras, Buena Vista and Miraflores towns consume. This proyect shows problems of health which arsenic causes in the poblation, the theortic average's increment that a person ingest, the use of recycled aluminium to have aluminum sulfate for using to clot and precipitate the arsenic and the extraction abtained for this component. Also is propose an action plan to the region with the purpose of doing better the environment's quality.
\end{abstract}

\section{INTRODUCCIÓN}

El agua es primordial conservarla y mantenerla libre de contaminación antropogénica; sin embargo, la composición geomorfológica en diferentes regiones del planeta determina la presencia en el agua subterránea y superficial de varios elementos y compuestos inorgánicos potencialmente nocivos para el ser humano, como es el caso del arsénico. Existen regiones en nuestro país donde por las caracteristicas geológicas sus acuíferos contienen arsénico y otros metales disueltos en el agua. Estos elementos constituyen parte de una gran cantidad de minerales; $y$, naturalmente, a través de procesos geoquímicos las sales que contienen arsénico se solubilizan en los acuiferos [1,2]. Este problema del hidroarsenicismo de origen geológico-sedimentario no es nuevo y afecta en varios lugares en el mundo (Tucumán, Argentina, región norte de Chile, México, Tacna, Perú, etc.).

\subsection{Límites máximos permisibles:}

El arsénico, aun en concentraciones muy bajas, puede causar a largo plazo enfermedades neoplásicas malignas, queratosis palmoplantar, atrofia cutánea, conjuntivitis, astenia o trastornos neurológicos y hematológicos, entre otros, lo que justifica la decisión de la Organización Mundial de la Salud (OMS) de reducir cinco veces el límite máximo admisible: de 0,05 a 0,01 ppm (partes por millón) $[3,4]$.

Dentro de la legislación ambiental vigente, existen varias normas que establecen los niveles máximos permitidos de contenido de arsénico en agua (ver Tabla 1). Las tendencias actuales en paises altamente industrializados proponen límites máximos de contenido de arsénico que deberian ser de hasta varios cientos de veces inferiores a los niveles actuales. Se ha calculado que al nivel de $0,05 \mathrm{mg} / \mathrm{l}$ de arsénico, establecido como norma en varios paises, el riesgo de morir de cáncer de hígado, pulmón, riñón o vejiga causado por beber 1 litro de agua diario durante toda la vida podría ser de 13 por cada 1000 personas expuestas.

TABLA 1. Normas que Establecen los Niveles Máximos Permitidos de Contenido de Arsénico en Agua

\begin{tabular}{|l|c|}
\hline \multicolumn{1}{|c|}{ Norma Actual } & $\begin{array}{c}\text { Concentración } \\
\text { de Arsénico }\end{array}$ \\
\hline $\begin{array}{c}\text { Norma de la Organización Mundial } \\
\text { de la Salud (OMS) }\end{array}$ & $0,010 \mathrm{mg} /$ \\
\hline Norma Mexicana & $0,050 \mathrm{mg} /$ \\
\hline Norma Chilena & $0,050 \mathrm{mg} /$ \\
\hline Norma en Estados Unidos & $0,050 \mathrm{mg} /$ \\
\hline Norma Argentina & $0,050 \mathrm{mg} /$ \\
\hline Norma Francesa & $0,050 \mathrm{mg} /$ \\
\hline Norma Alemana & $0,010 \mathrm{mg} /$ \\
\hline Comunidad Económica Europea & $0,050 \mathrm{mg} /$ \\
\hline Norma Peruana & $0,1 \mathrm{mg} /$ \\
\hline
\end{tabular}

1.2. Tecnologías para reducir el arsénico del agua

A lo largo del tiempo se han ensayado diferentes técnicas para reducir el arsénico del agua, entre ellas [1-8]: 
1. Destilación solar

2. Adsorción- coprecipitación usando sales de hierro y aluminio

3. Adsorción en alúmina activada / carbón activado / bauxita activada

4. Ósmosis inversa

5. Intercambio iónico

6. Oxidación seguida de filtración

7. Ablandamiento con cal

El $\mathrm{As}^{5+}$ puede ser removido en forma efectiva por coagulación con sulfato de aluminio o hierro y por los procesos de ablandamiento con cal y soda. Los coagulantes señalados se hidrolizan formando hidróxidos, sobre los cuales el $\mathrm{As}^{+5}$ se adsorbe y coprecipita con otros iones metálicos y las eficiencias de remoción se muestran en Tabla 2:

TABLA 2. Eficiencia de Coagulantes en la Remoción de Arsénico

\begin{tabular}{|c|c|c|}
\hline \multirow{2}{*}{ COAGULANTE } & ARSENATO, $\mathrm{As}^{+5}$ & ARSENITOAs ${ }^{+3}$ \\
\hline & REMOCIÓN (\%) PH & REMOCIÓN (\%)PH \\
\hline SULFATO FÉRRICO Fe $\left(\mathrm{SO}_{4}\right)$ & $100<9.0$ & $20<9.0$ \\
\hline SULFATO DE ALUMINAAI ${ }_{2}\left(\mathrm{SO}_{4}\right)$ & $90<7.0$ & $50<7.0$ \\
\hline
\end{tabular}

La remoción de arsénico con procesos convencionales de coagulación con sales de aluminio y hierro y ablandamiento con soda son dependientes del $\mathrm{pH}$ del agua tratada, del tipo y dosis de coagulante asi como de la concentración inicial de este elemento.

\section{OBJETIVOS}

1. Análisis del estado actual de la calidad del agua y regulación ambiental del arsénico de acuerdo con diferentes normas y guías de calidad de agua potable.

2. Alternativa de solución mediante coagulación del arsénico por el sulfato de aluminio.

3. Grado de exposición de la población según niveles de concentración de arsénico en el agua distribuida.

\section{ANTECEDENTES}

El distrito de Sama se encuentra en la provincia de Tacna, región Tacna, a una altura (capital) de 374 m.s.n.m. cuya población se estima en 2494 con una superficie $\left(\mathrm{km}^{2}\right)$ de 1115,98 y representa el 2,2 en densidad poblacional $\left(\mathrm{Hab} / \mathrm{km}^{2}\right)$. La población estimada en la capital (Las Yaras), Buena Vista y Miraflores es de 950 y que son los lugares que utilizan el agua proveniente de un manantial vía distribución por tubería que está ubicado a $5 \mathrm{~km}$ y aproximadamente extrae un caudal de $40 \mathrm{l} / \mathrm{min}$.

El arsénico encontrado en el agua de acuerdo con los estudios realizados en el año 1999 (agosto), agua superficial en el puente; 2001 (junio) con respecto al río Sama por DIGESA $[9,10]$ fueron los siguientes (Tabla No. 3 ):

TABLA 3 Parámetros Determinados en Calidad de Agua en el distrito de Sama.

\begin{tabular}{|l|r|c|c|}
\hline \multicolumn{1}{|c|}{ Parámetro } & \multicolumn{3}{c|}{ Fecha } \\
\hline & Junio 1999 & \multicolumn{1}{c|}{ Febrero, 2001 } & Agosto 2001 \\
\hline $\mathrm{pH}$ & 7,93 & 7,21 & - \\
\hline Temperatura $\left({ }^{\circ} \mathrm{C}\right)$ & 17,80 & 20,1 & - \\
\hline Conductividad $(\mu \mathrm{S} / \mathrm{cm})$ & 800 & 3980 & - \\
\hline Sól. Tot. Dis. $(\mathrm{mg} /)$ & 480 & 2000 & - \\
\hline Nitratos $(\mathrm{mgl})$ & 0,60 & -- & - \\
\hline DBO $(\mathrm{mg} / \mathrm{l})$ & 0 & - & - \\
\hline Colif. Total/100 $\mathrm{ml}$ & $9,7 \times 10^{3}$ & - & - \\
\hline Coliforme fecal/100 $\mathrm{ml}$ & $<1$ & - & - \\
\hline Arsénico $(\mu \mathrm{g} /)$ & 0,0602 & 0,075 & 0,01 \\
\hline Cadmio $(\mu \mathrm{g} / \mathrm{l})$ & 0,01 & 0,01 & $<0,003$ \\
\hline Cromo $(\mu \mathrm{g} / \mathrm{l})$ & 0,005 & $<0,050$ & $<0,010$ \\
\hline Mercurio $(\mu \mathrm{g} / \mathrm{l})$ & -- & - & $<0,01$ \\
\hline Plomo $(\mu \mathrm{g} / \mathrm{l} /)$ & 0,130 & $<0,025$ & $>0,005$ \\
\hline
\end{tabular}

Fuente: DIGESA, varios periodos

\section{EXPERIMENTACIÓN Y ANÁLISIS}

Desde el punto de vista tecnológico, los resultados de la investigación contribuirán a conocer uno de los métodos utilizando residuos de aluminio mediante el reciclado para obtener un gel que luego se aplicará al agua para ver su efecto; y, desde el punto de vista práctico, el estudio servirá para identificar efectivamente, las causas de la contaminación, posible tratamiento para establecer la política de gestión para su uso o definitivamente descartar la calidad de agua que consume la población de Sama.

\subsection{Caracterización de las Aguas: Análisis}

Se realizó toma de muestras de agua, en la captación y en la salida de un caño para uso domiciliario y su posterior análisis. Se codificaron las muestras como:

- Muestra $01,03=$ captación de agua.

- Muestra 02, 04 = agua de consumo

La caracterización de metales pesados (As, $\mathrm{Cd}, \mathrm{Fe}$, etc.) no se realizó.

Con los resultados obtenidos se han establecido comparaciones con la reglamentación peruana para agua de consumo humano y valores y con la guia para agua potable de la Organización Mundial de la Salud (OMS).

Los valores obtenidos son los siguientes mostrados en Tabla No. 4: 
TABLA 4. Análisis del agua de Consumo del Distrito de Sama Las Yaras y Comparación con los Límites Máximos Permisibles (LPM).

\begin{tabular}{|c|c|c|c|c|c|c|}
\hline \multirow[t]{2}{*}{ Parámetros } & \multicolumn{2}{|c|}{$\begin{array}{l}\text { Muestra } \\
(3 / 09 / 05)\end{array}$} & \multicolumn{2}{|c|}{$\begin{array}{l}\text { Muestra } \\
(09 / 12105)\end{array}$} & \multirow[t]{2}{*}{$\begin{array}{l}\text { R. Peruano de } \\
\text { agua potable }\end{array}$} & \multirow[t]{2}{*}{$\begin{array}{l}\text { Guia de } \\
\text { O.M.S. }\end{array}$} \\
\hline & 01 & 02 & 03 & 04 & & \\
\hline $\mathrm{pH}$ a $17^{\circ} \mathrm{C}$ & 6,5 & 6,7 & 6,55 & 6,6 & 10 & $6,5-8,5$ \\
\hline Conductividad (umhos $/ \mathrm{cm}$ ) & 2900 & 900 & 2900 & 1050 & $=$ & - \\
\hline Dureza & 1400 & 1350 & 1100 & 1000 & - & 500 \\
\hline Sólidos totales & 1900 & 1300 & 1750 & 1000 & 1000 & 1000 \\
\hline Coliformes totales $/ \mathrm{ml}$ & & & 900 & 40 & $2 \times 10^{4}$ & \\
\hline As (mgl) & - & - & 0,085 & 0,043 & 0,1 & 0,01 \\
\hline
\end{tabular}

$\left(^{*}\right)$ Ley General de Aguas 17752 Clase II, limites máximos permisibles, en U. F. C. (Unidades formadoras de colonias).

La dureza y sólidos totales disueltos para ambas muestras están en mayores niveles que los recomendados por la OMS, y estos parámetros son los que influyen en la calidad organoléptica del agua. La conductividad es un parámetro alto en ambas y este parámetro tiene relación con la dureza que tienen las aguas de captación y para consumo. Los coliformes fecales totales se encuentran por debajo de los niveles especificados por la norma peruana. El contenido de arsénico supera en 8 y 4 veces la norma de la OMS.

\subsection{Consideraciones para la Eliminación del} As

\section{a). Selección de los Productos:}

Oxidante: En la introducción se indicó la conveniencia de la oxidación del $\mathrm{As}^{+3}$, que predomina en las aguas subterráneas antes del tratamiento con coagulación. Debido a que los arsenitos se oxidan fácilmente a arsenatos con cloro, se seleccionó el hipoclorito de calcio al $70 \%$ de cloro activo como material oxidante.

Coagulantes: El coagulante seleccionado para la prueba fue el sulfato de aluminio (a partir de aluminio recuperado de residuos) debido a que es conocido que los arsenatos se precipitan o se adsorben sobre los hidróxidos de hierro y aluminio mediante reacciones superficiales específicas.

Previamente a la oxidación, el aluminio se cortó en trozos pequeños, se agregó ácido sulfúrico $1 \mathrm{M}$ y luego se filtró y lavó, previamente las latas de aluminio se limpiaron. El filtrado, lavado y secado se utilizaron para realizar la prueba de coagulación.

En la Tabla 5 se muestran datos del análisis de agua antes y después del experimento.
TABLA 5. Análisis físico-quimicos del agua de consumo del distrito de Sama Las Yaras, antes y después de tratamiento. Fecha: viernes 26 de marzo del 2006.

\begin{tabular}{|c|c|c|c|c|}
\hline \multirow{2}{*}{ Parámetros } & \multicolumn{2}{|c|}{ Muestra } & \multirow{2}{*}{$\begin{array}{l}\text { Reglamento } \\
\text { Peruano de } \\
\text { agua potable }\end{array}$} & \multirow{2}{*}{$\begin{array}{l}\text { Guia de la } \\
\text { O.M.S. }\end{array}$} \\
\hline & Antes & Después & & \\
\hline $\mathrm{PH}$ a $26,59^{\circ} \mathrm{C}$ & 7,02 & 7,45 & 10 & $6,5-8,5$ \\
\hline Conductividad (umhos/cm) & 3945 & 1300 & - & - \\
\hline Dureza & 1100 & 500 & - & 500 \\
\hline Sólidos totales & 1850 & 900 & 1000 & 1000 \\
\hline $\mathrm{As}(\mathrm{mg} / \mathrm{l})$ & 0,043 & 0,025 & 0,1 & 0,01 \\
\hline
\end{tabular}

Como se observa, la remoción ha sido del $44,44 \%$, un parámetro todavia 2,5 veces por encima del valor de la OMS. Esto es debido a que el coagulante (sulfato de aluminio) no cumplió correctamente los objetivos como tal, ya que la efectividad teórica es del $95 \%$. En todo caso los otros parámetros se han reducido considerablemente, y es conveniente realizar otros experimentos controlando en mayor detalle las variables y el entorno, como también las características del análisis.

\subsection{Análisis de Riesgo [4]}

Para definir el riesgo por exposición al arsénico, se siguió parte de la metodología del análisis de riesgo, procedimiento que se lleva a cabo antes de decidir qué acciones se tomarán para reducir o eliminar un peligro potencial para la salud de la población.

\section{Dosis de Exposición:}

Es necesario establecer la dosis probable de exposición a este contaminante en el agua de bebida. Se utilizan las dosis de referencia a partir de las cuales se observan efectos adversos y refieren la exposición a una concentración umbral o al limite de la concentración Más Baja de Observación de Efectos Adversos, LOAEL, por sus siglas en inglés.

\section{Dosis de Referencia del Arsénico:}

La dosis de referencia LOAEL para el arsénico se calcula a partir del valor calculado en $0,043 \mathrm{mg} / \mathrm{l}$ de arsénico en el agua. En la fórmula se incluye una estimación de la ingestión de arsénico a través de los alimentos de 0,002 mg/dia, un consumo de 4,5 litros de agua al día y un peso corporal promedio de $55 \mathrm{~kg}$ en las personas expuestas. 
$L O A E L=[(0,043 \mathrm{mg} / \mathrm{l} \times 4,5 \mathrm{l} / \mathrm{d})+0,002 \mathrm{mg} / \mathrm{d}] / 55$ $\mathrm{kg}=0,0036 \mathrm{mg} / \mathrm{kg}$-dia.

Para calcular el riesgo en la población, en este análisis se utilizó la fórmula siguiente [4]:

$$
g(d)=\left(0.564398 \times 10^{-8}\right) d+\left(0.435613 \times 10^{-9}\right) d^{2}
$$

Donde: $d=$ dosis de exposición

El riesgo obtenido es el siguiente:

$2,0324 \times 10^{-11}$ valor relativamente alto de acuerdo con referencias bibliográficas [4].

El método puede resolver en forma sencilla y económica el problema de las aguas arsenicales. Además, se puede suponer que el sulfato de aluminio también puede adsorber otros metales disueltos en el agua, como el cromo, el molibdeno y otros. Pero es conveniente realizar mayores ensayos bajo consideraciones de un presupuesto que permita realizar los análisis respectivos.

\section{CONCLUSIONES:}

1. Las alternativas de tratamiento propuestas en laboratorio (sulfato de aluminio) logró remover nivel de arsénico hasta un $44 \%$.

2. Es posible usar aluminio recuperado de latas u otro componente eliminado como residuo con la finalidad de reducir el consumo de metal en origen.

3. El mecanismo de remoción del arsénico probablemente sea una combinación de interacciones físicas y químicas entre las que debe predominar la adsorción y coprecipitación de los arsenatos.

\section{RECOMENDACIONES}

Estudiar a nivel de laboratorio la posibilidad de utilizar varios componentes de mezclas del coagulante a usarse y otros (cloruro de fierro, gel de aluminio, etc.). De preferencia materiales locales o nacionales y en lo posible reciclados.

- Se debe probar la reproducibilidad in situ de la eficiencia de remoción del arsénico antes de darle uso poblacional.

- La adopción de límites de calidad de agua a parámetros actuales y que es de urgencia en nuestro pais por las entidades que tienen que velar por la salud de la población, a partir de valores guías propuestos por la OMS. Estos valores permitirán a las autoridades y organismos de control la revisión, actualización o adopción de límites en agua de bebida, asumiendo riesgos aceptables para diferentes tiempos de exposición compatibles con las realidades hidrogeológicas y socioeconómicas de la región.

- Elaborar mapas ambientales de riesgo según las concentraciones de arsénico de toda la cuenca del valle y a una escala de colores para diferenciar las áreas con diferentes concentraciones promedio de arsénico.

\section{BIBLIOGRAFIA}

[1] Luján, Juan Carlos, "Un hidrogel de hidróxido de aluminio para eliminar el arsénico del agua", Rev. Panam. Salud Publica, vol.9, no.5, Washington, 2001: 302-305.

[2] Nicolli, H. et. al . "Geoquímica del arsénico y de otros oligoelementos en aguas subterráneas en la llanura suroriental de la provincia de Córdova, s/d" [en linea], www.bibliotecas.unc.edu.ar/cgibin/LibreoOPAC?accionbuscar\&expresionARSE NICO> 1985,p:112.

[3] AJE, "Arsenic Ingestion and Internal cancers" A Review. American Journal of Epidemiology, Vol 139, No $12 ; 1994$.

[4] Vega G., Sylvia, "Riesgo sanitario ambiental por la presencia de arsénico y fluoruros en los acuiferos de $M$ é $x$ i con [ e n li n e a ] . $<$ bibliotecnica.upc.es/PFC/arxius/migrats/411511.pdf $->$ [consulta,13-03-05].

[5] Imta. "Remoción de arsénico de agua para consumo humano". Informe Final. México, diciembre 1997.

[6] Sancha, A. M. "Experiencia chilena en abatimiento de arsénico. Seminario Potabilización de Aguas con Arsénico y Flúor". $9^{\circ}$ Congreso Argentino de Saneamiento y Medio Ambiente. Córdoba-Argentina, junio, 1996.

[7] Callegaro, R. S. et. al... Métodos prácticos para la corrección de aguas arsenales. Dpto de Hidráulica, Facultad de Ingeniería, Universidad Nacional de la Plata, 1998.

[8] Essan: "El camino para reducir notoriamente el arsénico en el agua potable", José A. Granada M., Walter E. Cerda A., David A. Godoy A., Revista AIDISChile, dic. 2003, pág 44-49.

[9] DIGESA, Resultados de análisis realizados a las muestras del río Sama y confluencias. 19 y 20 de agosto de 1999.

[10] DIGESA, Interpretación de resultados DIRSA Tacna Las Yaras, mayo 2001. 